\title{
La guerre d'Espagne dans la presse impériale
} (1808-1814)

Jean-René Aymes

\section{(2) OpenEdition \\ 12 Journals}

\section{Édition électronique}

URL : https://journals.openedition.org/ahrf/1697

DOI : 10.4000/ahrf.1697

ISSN : 1952-403X

Éditeur :

Armand Colin, Société des études robespierristes

\section{Édition imprimée}

Date de publication : 1 juin 2004

Pagination : 129-145

ISSN : 0003-4436

\section{Référence électronique}

Jean-René Aymes, «La guerre d'Espagne dans la presse impériale (1808-1814) », Annales historiques de la Révolution française [En ligne], 336 | avril-juin 2004, mis en ligne le 15 juillet 2007, consulté le 23 avril 2022. URL : http://journals.openedition.org/ahrf/1697 ; DOI : https://doi.org/10.4000/ahrf.1697

Ce document a été généré automatiquement le 23 avril 2022.

Tous droits réservés 


\title{
La guerre d'Espagne dans la presse impériale (1808-1814)
}

\author{
Jean-René Aymes
}

1 Une étude de l'image "à chaud" de la Guerre d'Indépendance en France, c'est-à-dire une étude de la vision lacunaire et déformée qu'en eurent les sujets de Napoléon, devrait incorporer, outre la presse impériale seule examinée ici $^{1}$, les Mémoires autobiographiques des acteurs et des témoins ${ }^{2}$, et les nombreux rapports et mémoires accessibles dans divers fonds d'archives parisiennes. Il faudrait y adjoindre les ouvrages, peu nombreux et en général décevants, où sont décrits les épisodes majeurs de la guerre à peine achevée ${ }^{3}$. Enfin, en se plaçant aux frontières de l'historiographie, il faudrait inclure, dans la mesure où l'on demeure dans le champ de l'image ici altérée, pittoresque, plaisante..., un certain nombre de comédies, de vaudevilles, de pièces équestres... qui évoquent l'Espagne plongée dans la guerre, sous le titre, par exemple, de La Belle Espagnole ou l'entrée triomphale des Français à Madrid (Paris, 1809).

La presse : statut et traitement du matériau

2 Il est connu que la presse des années 1808-1814, qualifiable de "presse d'État ", a atteint un haut degré dans la «désinformation ». Il serait oiseux d'exposer ici, après André Cabanis ${ }^{4}$ et Remedios Solano Rodríguez, les objectifs de l'empereur et les méthodes tendant à obtenir que toutes les informations relatives à la guerre d'Espagne soient contrôlées et, parfois, élaborées par lui.

3 Le nombre des périodiques survivants après 1811 fait illusion : quatre à Paris et un par département. En effet, les responsables du Journal de l'Empire, du Journal de Paris et du Journal des Débats ne peuvent s'écarter un tant soit peu de la version officielle que livre Le Moniteur ${ }^{5}$ et qui est la seule tolérée par les censeurs. Alors qu'il se trouve à Bayonne en mai 1808, Napoléon n'hésite pas à écrire à Fouché, son Ministre de la Police Générale :

«Il faut tenir la main à ce qu'aucun journal ne parle des affaires [...] de l'Espagne qu'après Le Moniteur. $»^{6}$

4 Les informations relatives à la guerre d'Espagne procèdent des «Bulletins des Armées d'Espagne ", des rapports particuliers, parfois longs et détaillés, envoyés par les 
généraux, des lettres interceptées (probablement imaginaires) trouvées sur des Espagnols ou des Anglais capturés, et enfin - chose plus inattendue- des longs extraits (dont l'authenticité est sujette à caution) de journaux anglais (The Star, The Courier) qui parfois rendent compte des débats tenus à la Chambre des Communes lorsque les affaires d'Espagne y sont évoquées.

Grâce à cette liberté d'expression qui faisait défaut dans la France du Premier Empire, les Anglais avaient tout loisir pour déplorer les revers occasionnels des troupes britanniques, pour critiquer certaines erreurs commises par des responsables et pour dévoiler les objectifs poursuivis par les autorités londoniennes au sud des Pyrénées. Pour donner une impression de véracité, les rédacteurs ${ }^{7}$ des journaux de l'Empire ont parfois l'habileté d'introduire, en notes de bas d'articles, des commentaires, soit rectificateurs, soit sarcastiques et chargés de rappeler que les militaires et les journalistes anglais exagèrent ou, pis encore, mentent sans vergogne! Il faudrait prendre la peine de confronter les pièces originales et la version française correspondante pour apprécier la part faite à l'addition, l'amputation, l'amplification.

Ces trois termes sont applicables, du reste, au traitement que subissent la plupart des informations relatives à la guerre d'Espagne, qu'elles proviennent de la Péninsule, de l'Amérique espagnole, d'Angleterre ou de France.

7 On demeure confondu par le caractère voyant et peu sophistiqué des procédés employés pour abuser l'opinion publique française au moyen de mensonges éhontés, de manipulations et d'occultations. Tout se passe comme si les lecteurs étaient tenus pour des individus niais, dépourvus de mémoire et de jugeote.

Les zones d'ombre

8 Au premier rang des événements militaires déplorables que les journalistes doivent masquer le plus longtemps possible figure la honteuse capitulation de Baylen (22 juillet 1808). Le 2 août, de vagues considérations générales donnent à penser que les Anglais, plutôt que les Espagnols, ont remporté des victoires, non précisées. Au début de septembre seulement, le $M U$ laisse deviner la gravité de la défaite de Baylen :

"Cette nouvelle inattendue a donné de l'audace aux insurgés. »

9 Mais il est exclu que soit altérée l'image prestigieuse de l'armée impériale en dévoilant qu'elle a déposé les armes devant une troupe espagnole composite. Toute la responsabilité retombera sur Dupont dont la conduite a été contraire à tous les principes et qui n'a pas su faire montre de "courage civil», ni d'habileté pendant la négociation (MU, 5 septembre 1808). Les lecteurs ne sauront à peu près rien de la bataille elle-même qui - dirait-on - n' a eu ni vainqueurs (car non désignés) ni vaincus (hormis Dupont).

10 On compterait sur les doigts de la main les allusions aux fautes commises par les militaires français, toujours exemplaires par leur vaillance et leur habileté. Tout juste saura-t-on, à la fin de 1811, que le duc de Dalmatie est mécontent du général Reynaud, commandant à Ciudad Rodrigo, qui, ayant tenté une sortie imprudente, est tombé aux mains d'un escadron de la «bande de don Julian » (MU, 11 décembre 1811).

11 Comme si ces maréchaux et ces généraux avaient reçu la mission exclusive d'anéantir les troupes anglaises et de disperser les bandes de brigands, les journalistes s'arrêtent rarement sur leurs tâches d'administrateurs des territoires plus ou moins contrôlés par eux. Seule, se détache la figure de Suchet qui, après l'épouvantable siège subi par Saragosse, a à cœur de nettoyer la ville, de faire respecter « les propriétés, la religion et 
les coutumes du pays » et de rétablir le cours normal de la vie collective, par exemple en ordonnant la reprise des courses de taureaux auxquelles il se rend en personne (MU, 8 novembre 1809). En 1812, son œuvre louable de pacification constructive semble porter ses fruits en Catalogne :

«Le Catalan revient de ses préventions, les torches du fanatisme s'éteignent [...].

L'Ampurdan tout entier est soumis. » (JE, 24 juin 1812)

12 De façon globale, les sujets militaires l'emportent largement sur les sujets politiques, sauf au cours des deux premières années (1808 et 1809) où tout paraît encore possible en matière de réorganisation de l'État espagnol. En 1808, on informe le public sur les intentions de l'empereur, on rend compte des mesures concrètes prises par le gouvernement de Joseph, en se gardant de préciser - et pour cause - si elles reçoivent, ou non, un début d'application. En revanche, au cours des années suivantes, en raison de la mauvaise tournure prise par les événements, les perspectives politiques s'estompent, puis s'effacent ; il serait incongru de rappeler que Napoléon se voulait - le veut-il encore ? - le "régénérateur de l'Espagne »; la belle œuvre de Suchet est perdue de vue ; désormais, l'attention est portée exclusivement sur les efforts déployés par les militaires. On dirait que l'affaire espagnole a cessé d'être politique.

Alors que la guerre d'Espagne comporte, à l'évidence, des enjeux économiques que les historiens, notamment les marxistes, ont mis en valeur, la presse impériale n'aborde pas ce sujet, sauf à répéter, de façon catégorique, que les Anglais, ces "grands spéculateurs ", ont fait le projet de s'emparer du Portugal, puis de s'installer en Espagne pour y servir leurs intérêts égoïstes :

"L'insurrection des Alpujarras intéressait les Anglais, non seulement pour maintenir l'esprit de révolte, mais aussi pour pouvoir tirer les plombs que les mines de ces montagnes produisent. » (MU, 17 juillet 1810)

En ce qui concerne le côté français, la surprise pour l'observateur d'aujourd'hui vient de l'importance attribuée à un seul produit ibérique : la fameuse laine des moutons mérinos $^{8}$. Première allusion dans le JE du 28 novembre 1808 :

«On a trouvé à Saint-Ander (Santander) un dépôt considérable de laines, qui est transporté en France. »

Puis le MU du 17 mars annonce la vente prochaine, à Bayonne, de 2500 balles de différentes catégories. À l'occasion d'une nouvelle vente, le journal consacre une pleine page à la reproduction des «marques " et «contremarques». En 1810, une nouvelle grande vente a lieu à Bayonne et une autre à Paris, au Conservatoire des Arts et Métiers ( $M U, 14$ mars 1810). Alors que les affaires vont de mal en pis en 1813, le $M U$ annonce une vente, avec "grande affluence de monde », de "laines et bêtes à laine fine d'Espagne, dans l'établissement impérial et rural de Rambouillet» (MU, 8 juillet 1813). Naturellement, on aura soin de dissimuler que ces laines, prétendument confisquées aux insurgés, n'ont pas toutes été mises aux enchères et aussi que tel général, après s'être approprié d'imposants troupeaux, les a fait conduire sur ses terres, en France...

Le socle des invariants

16 En opposition avec l'évolution signalée plus haut, qui conduit à asseoir progressivement la prééminence du militaire sur le politique, l'interprétation de la guerre d'Espagne repose en même temps sur une série d'invariants qui sont, soit des postulats formulés au début du conflit, soit des stéréotypes parfois vieux de plusieurs siècles. 
17 L'un de ces clichés se rattache à la «Légende noire » de l'Espagne. On réactualise l'idée que les Espagnols sont paresseux, ignorants, veules, etc. Le comportement des "brigands ", lorsqu'ils combattent contre les soldats français, en fournit l'illustration réitérée. Il faut observer toutefois que, entre 1807 et l'été 1808 ("Dos de mayo» madrilène et Baylen), l'image péjorative des Espagnols avait été partiellement corrigée, dans la mesure où Napoléon et ses maréchaux se faisaient fort de pouvoir compter sur le soutien de la fraction éclairée de la population.

Mais cette rectification n'est jamais appliquée à l'armée régulière espagnole qui, malgré le récent démenti apporté par Baylen, inspire des opinions extraordinairement méprisantes :

«À la vérité et sans faire un tort à la bravoure de nos soldats, on doit dire qu'il n'y a pas de plus mauvaises troupes que les troupes espagnoles; elles peuvent, comme les Arabes, tenir derrière des maisons; mais elles n'ont aucune discipline, aucune connaissance des manoeuvres, et il leur est impossible de résister sur un champ de bataille. Les montagnes mêmes ne leur ont offert qu'une faible protection. Mais grâce à la puissance de l'Inquisition, à l'influence des moines et à leur adresse à s'emparer de toutes les plumes et à faire parler toutes les langues, on croit encore dans une grande partie de l'Espagne que Blake a été vainqueur, que l'armée française a été détruite, que la garde impériale a été prise. » (MU, 4 décembre 1808)

Ce paragraphe réunit deux des composantes de ce socle d'invariants appliqués à la Guerre d'Indépendance : d'une part, on retrouve ce réflexe, idéologique et discursif, qui conduit à ramener l'Espagne au rang de l'Égypte (à la même époque, on dit des paysans espagnols qu'» on ne peut les comparer qu'aux fellahs d'Égypte »); d'autre part, on tiendra pour avéré, jusqu'à la fin du conflit, que l'opinion publique espagnole est façonnée, en profondeur, par l'Inquisition, et, concrètement, par le clergé régulier. L'armée elle-même est gâtée par cette double et odieuse mainmise :

« Ignorance crasse, folle présomption, cruauté contre le faible, souplesse et lâcheté avec le fort: voilà le spectacle que nous avons devant les yeux. Les moines et l'inquisition ont abruti cette nation.» ( $M U, 22$ novembre 1808, "4e Bulletin de l'Armée d'Espagne »)

La longue «Relation des événements d'Espagne ", proposée aux lecteurs du MU, le 5 septembre 1808, offre la quintessence - si on peut dire - de cette «Légende noire » à laquelle appartient ce véhément anticléricalisme :

«Les moines, presque partout sans aucune distinction, et fanatiques au plus haut degré, exercent une puissante influence sur les classes inférieures du peuple, qui vivent dans une ignorance plus entière en Espagne que partout ailleurs, et qui, sous une telle direction, n'ont fait de progrès depuis plus d'un siècle que dans le goût des pratiques superstitieuses et de l'oisiveté. »

21 On se doute que les adversaires anglais et espagnols sont constamment disqualifiés. À ne lire que la presse, on ne voit pas qui des soldats anglais ou des insurgés espagnols inspirent le plus de haine. Entrevus dans le $M U$, les soldats de S. M. Britannique pillent demeures, églises et couvents, maltraitent les indigènes, massacrent les prisonniers français, se croient en pays conquis ; «ils font verser le sang espagnol pour nourrir le monopole anglais ». Cette vision très péjorative des soldats anglais sera corrigée après la chute de l'Empire, dans plusieurs Mémoires autobiographiques d'anciens soldats français qui, au lieu d'exécrer ces adversaires pour leur sauvagerie ou leur bassesse, tiendront à révéler que, ici et là, ils eurent affaire à des Anglais magnanimes, ou compatissants, ou ayant conservé le sens de l'honneur. Mais, tant que dure la guerre, l'anglophobie est virulente et sans nuances; elle vise plus encore les dirigeants que la 
population. Il est entendu que les Anglais, en raison de leur convoitise et de leur volonté hégémonique, sont les responsables de la guerre, puisqu'ils prétendent s'établir au Portugal, dominer l'Espagne et s'emparer de ses colonies d'Amérique. Tant d'abus et de vilenie imputés aux Anglais ne peuvent qu'indisposer les Espagnols ainsi portés à craindre et détester de si méchants alliés (MU, 10 janvier 1809). Un autre concept appartient au « socle des invariants » : celui de «mésentente » ou de « désunion » qui caractérise le camp des adversaires, alors que (contre toute vérité) le camp français serait celui de l'unité de vues et de la collaboration harmonieuse.

Comme il est postulé que les peuples latins sont faits pour s'entendre, on veut persuader les lecteurs que les Anglais et les Espagnols ne pourront jamais s'accorder :

«La différence de la langue, des mœurs et de la religion n'a pas peu contribué à

cette disposition des esprits. » (MU, 8 janvier 1809)

La mésentente sévit aussi, en 1812, dans le camp espagnol, comme le dévoile une lettre (authentique ?) rendant compte des désaccords entre, d'un côté, Ballesteros et, de l'autre, le duc de l'Infantado et Carlos O'Donnell (JE, 22 juin 1812).

Les rapports ne seraient pas davantage harmonieux entre les responsables militaires espagnols et les autorités civiles, à l'exemple du différend qui aurait surgi en juin 1809 entre le général «La Romana » et la Junte d'Oviedo qu'il aurait finalement dissoute après avoir proclamé que tous ses membres étaient « des ignorants, des imbéciles et des hommes corrompus » (MU, 12 juin 1809).

En 1812, contredisant ce que les historiens ont établi, à savoir que les "partidas » (les petites troupes de guérilleros) auraient plutôt tendance à grossir et à se réunir, et ce à la demande des autorités supérieures, le JE fait état de la désunion qui s'aggrave au sein des «bandes de brigands » et qui les amène à se scinder et, parfois, à se réduire à des groupes de quatre ou cinq hommes (JE, 7 mai 1812).

La « guerre classique»

Les «Bulletins des Armées d'Espagne » et les rapports particuliers des maréchaux et des généraux sont les principaux documents qui livrent des informations qualifiables de traditionnelles, relatives aux grands combats et aux sièges de villes, avec déroulement des opérations, nom des unités engagées, nombre de morts, de blessés et de prisonniers, perspectives ouvertes...

28 Le second siège de Saragosse est l'épisode militaire auquel la presse impériale accorde le plus d'importance, plus encore symbolique qu'au regard de la stratégie, parce qu'on estime que "Saragosse a été le véritable siège de l'insurrection de l'Espagne » (MU, 2 mars 1809).

29 Et, de fait, ce sont huit pages entières que le $M U$ consacre, ce jour-là, au siège de Saragosse et, plus précisément, au journal des attaques conduites, presque heure par heure, par le « Corps Impérial du Génie ».

30 En seconde position, mais loin derrière, vient le siège de Tarragone qui offre l'occasion au comte Suchet de faire le récit détaillé de ses opérations et d'annoncer la reddition de la ville, au terme d'un siège qui aura duré deux mois et provoqué la mort de 4000 hommes (MU, 9 juillet 1811).

31 Ne serait-ce qu'en raison de la présence de l'empereur sur le terrain, la victoire de Somosierra, à la fin de novembre 1808 , passe pour être - à juste titre, il est vrai - un événement majeur, puisqu'elle ouvrait la route de Madrid'. 
32 Péninsule, les victoires notables remportées par les troupes napoléoniennes ne donnent lieu que rarement à des célébrations emphatiques, au rebours de ce qui se passe pour les victoires remportées en Italie ou en Allemagne. Aussi faut-il considérer comme exceptionnelle l'information livrée par le JP du 18 décembre 1808, relative à l'accueil que les Bordelais réservent à l' " heureuse nouvelle authentique » (sic) de la victoire de Somosierra (le nom n'apparaît pas) ; « M. le Préfet a bien voulu qu'on en instruisît, hier soir, le public au théâtre » :

«Le 30 novembre, l'ennemi a été culbuté dans ses positions; il a perdu toute son artillerie et tous ses caissons, et a été mis dans une déroute complète; son infanterie a été prise, tuée ou éparpillée. L'Empereur était à Buytrago [...].Tout porte à croire qu'il aura fait son entrée dans cette ville, le 2 décembre, jour anniversaire de son couronnement. Ces nouvelles ont été entendues hier, au spectacle, avec des transports de joie, et la salle a retenti des cris répétés: Vive l'Empereur!»

La guérilla

Pour ne pas donner à penser que la destitution de Ferdinand VII, puis les excès commis par les troupes françaises suscitent de l'indignation au sein de la population, les journaux dissimulent, jusqu'à l'automne 1808, la montée de la résistance. Cependant, déjà à travers le récit du soulèvement madrilène du 2 mai 1808 et des mesures de police rigoureuses qui s'ensuivent, les lecteurs français n'ignorent plus que l'adhésion unanime du peuple au nouveau pouvoir est devenue impensable, d'autant que l'agitation s'est étendue à plusieurs villes (Tolède, Valladolid, Palencia). Pour l'heure, hormis en ces lieux, la résistance semble plus rurale qu'urbaine. Elle est désignée habituellement par l'expression « rassemblements de paysans insurgés » qui nie tout à la fois leur motivation patriotique, la réalité d'une structuration en bandes organisées et l'ascension des leaders locaux au rang de «cabecillas ». S'il en est ainsi, les lecteurs français ne peuvent s'étonner, en 1808, que tous les affrontements entre ces «bandits » et les soldats napoléoniens tournent à l'avantage de ceux-ci qui n'ont aucun mal à provoquer la prompte dispersion de leurs piètres adversaires. Les lecteurs ne peuvent concevoir que cette dispersion relève peut-être d'une tactique adroite, commandée par l'inégalité des forces en présence. Toutefois, à l'automne 1808, une brève information méritait d'éveiller l'attention du public: du côté de Haro, le général Lasalle «a mis en pièces 3 ou 4000 insurgés qui avaient pris le nom de bataillon de litterarios " (JP, 2 novembre 1808), ce qui signifie que la guérilla ne recrute pas seulement parmi le prolétariat rural. Quelques semaines plus tard (JP, 20 novembre 1808), ressurgit l'allusion à un mouvement de résistance estudiantin, venu doubler le mouvement paysan placé sous l'égide de la religion : toujours du côté de Burgos, dans les poches des insurgés tués au combat, on a trouvé des papiers avec la mention de « compagnies de Brutus, compagnons del Popolo» (sic); et l'informateur d'ajouter : c'étaient des «compagnies des écoles»; d'autres bandes s'étaient données des «noms de saints: c'était l'insurrection des paysans». La substitution du terme « rassemblements» par celui de « compagnies » révèle que la guérilla se régularise, se structure, acquérant par là, aux yeux des adversaires français, un minimum de dignité et de légitimité.

u cours de l'année 1809, les autorités napoléoniennes et "joséphines " ne peuvent plus cacher que, de tous côtés, la guérilla s'étend et s'organise. Le 9 juillet, le $M U$ fait connaître que le roi Joseph en personne, flanqué d'un maréchal et de deux généraux, ne croit pas déroger à la majesté du trône en se lançant à la poursuite de la «bande de 
Vanegas»(Venegas). Le même jour, le journal consacre plusieurs lignes à la caractérisation - infamante, comme il se doit - de cette forme de résistance ; il s'agit, en l'occurrence, des bandes organisées par la Junte de Séville dans la région de Tolède :

«Leurs troupes les plus nombreuses, qui ne sont que de cent hommes, se composent de voleurs, de scélérats, antérieurement condamnés par les tribunaux, et formés depuis en compagnies de contrebandiers auxquels la Junte a donné des grades et dispensé des honneurs. Ce système est le même que celui mis, il y a plus de trois ans, en usage à Naples. »

35 À la même époque, est brandi un argument nouveau : loin de pouvoir compter sur la complicité ou l'appui des habitants, les « cabecillas » pratiquent à leur égard une forme d'oppression et de terrorisme. C'est ainsi que le général Bonnet juge le comportement des bandes du côté de Santander :

« Argent, vivres, troupeaux, ces bandits enlèvent tout aux propriétaires, aux curés mêmes et aux habitants les plus malaisés. Ceux qui voudraient opposer quelque résistance ou qu'ils croient d'un parti contraire au leur, ils les assassinent sans pitié. »

À la tête de ces " troupes assassines de Bandouliers ", figurent le curé Tapia connu pour ses excès dans les provinces de Valladolid et de Palencia », « un dominicain des Asturies revêtu de l'uniforme de capitaine » et " un abbé du même diocèse, nommé lieutenantcolonel»(MU, 21 juillet 1809). Les deux dernières précisions relatives aux grades qu'arborent les « cabecillas » signifient qu'est en cours un processus de rapprochement, au moins formel, entre l'armée irrégulière (celle de la guérilla) et l'armée régulière.

Bien que les récits des affrontements entre les soldats napoléoniens et les guérilleros aient tendance à maintenir ces derniers dans un anonymat collectif dévalorisant, on peut être surpris par le nombre relativement élevé des «cabecillas » nommément désignés, soit par leur patronyme, soit, le plus souvent, par leur nom d'emprunt : on a là les déjà fameux « Mina » (Espoz y Mina) et "El Empecinado », mais aussi «Urgate » (Ugarte), le curé Tapia, l'» alcade d'Otivar », «El Manco », «El Fraile», «El Médico », «Marquesito" ou «Marquesittos» (Díez Porlier), «Chaleco», «Tamborlan», «Barolucio », «Claros », « Comisario », « Tomasillo», « Rovira ", « Miralles", «Simonet », « Fabregas », « Saldivia », « Pessoduro » (« célèbre brigand, lieutenant de Mina $») .$.

38 À partir de 1810, se met en place et se diversifie tout un lexique qui rend compte de la montée en puissance et de l'élévation au rang de système et de tactique de cette guérillla spécifiquement espagnole et redoutable, bien que les comptes rendus de combats ne fassent état que de l'anéantissement ou de la dispersion des bandes. En 1811, le rapport émanant de l'Armée du Nord comporte l'expression inhabituelle «petite guerre » (MU, 8 juillet 1811). L'année précédente, les hommes du maréchal Augereau, gouverneur général de la Catalogne, avaient découvert la modalité régionale de la guérilla sous la forme des « Miquelets » et des « Soumatins » (« somatenes »).

Alors que, en 1808, les généraux servant en Andalousie déclaraient fièrement que les insurgés ne parvenaient même pas à se mettre à l'abri dans les sierras, quatre ans plus tard le général Clausel, qui se heurte à « Mina » (Francisco Espoz y Mina) dans la vallée navarraise du Roncal, reconnaît que son adversaire a toute facilité pour se défendre sur des hauteurs inaccessibles, dans des passages étroits, où « de faibles détachements sont en état d'arrêter de fortes colonnes ». 

l'expansion n'a pas été jugulée par les Français, a causé à ces derniers, mais aussi à la population, des dégâts considérables, comme l'indique le commentaire suivant, daté du 28 septembre 1813 :

«Ces bandes s'enrichissent par le pillage et obtiennent des succès faciles parce qu'elles n'attaquent presque jamais qu'à coup sûr [...]. Cette guerre intestine devient d'autant plus dangereuse que le nombre des guerillas s'accroît chaque jour par la désertion des troupes régulières qui n'ont d'autres rations qu'après qu'elles ont été distribuées aux Anglais. »

Plusieurs récits de combats menés contre les insurgés laissent entrevoir que les responsables militaires napoléoniens essaient de mettre en œuvre une tactique appropriée à cette forme inédite de lutte, qu'on ne qualifie pas encore de «contreguérilla ».

En 1810, les lecteurs du $M U$ apprennent, grâce à une lettre du général Decaen, que les Français ont organisé en Catalogne une réplique à la guérilla en mettant sur pied une petite troupe, très mobile, d'Espagnols collaborateurs des soldats français; il s'agit des «miquelets » (sic) de ce bientôt fameux Pujol, alias «Boquica », plus tard de sinistre mémoire :

«Les chasseurs de Lampourdan [sic], commandés par Poujol, et la compagnie de Gironne, tournèrent et enlevèrent la redoute de la Garriga; les Espagnols qui la défendaient s'enfuirent par la vallée de Figuère ; les chasseurs à cheval de Poujol les poursuivirent et en sabrèrent une vingtaine. » (MU, 20 novembre 1810)

Un autre moyen de combattre les «partis» ("partidas») consiste en la création de petites unités capables de se déplacer rapidement dans des secteurs escarpés. D'où la mention, en 1811, de "colonnes mobiles" qui entrent en action dans le nord de l'Espagne (MU, 8 juillet 1811) et d'une « compagnie de guides » du côté de Xérès en 1812 (JE, 4 février 1812).

À la suite du retournement de la situation militaire, lorsque les troupes angloespagnoles pénètrent en territoire français au début de 1814 et obligent les soldats napoléoniens à se mettre sur la défensive, les autorités napoléoniennes semblent découvrir - on peut en sourire - les vertus de cette guérilla que les Espagnols, sur leurs terres, avaient portée à un haut degré de terrible efficacité :

« Depuis que l'on a commencé à former des compagnies franches, tous les Basques se sont levés en masse et harcèlent l'armée anglaise de toute part. Ce genre de guerre convient également à la nature du pays et au caractère de ces actifs et intrépides montagnards. Accoutumés à manier avec adresse leurs fusils de chasse, ils tuent souvent les coureurs et les éclaireurs de l'ennemi. » (MU, 17 janvier 1814)

Les autorités espagnoles « rebelles »

Comme il s'agit de montrer, à des fins de propagande, que les "bandes d'insurgés " agissent de façon autonome et désordonnée, la réalité des Juntes provinciales et locales, dont certaines « partidas » dépendent, est pratiquement passée sous silence, alors que, somme toute, cette révélation eût pu servir à démontrer la dissolution du pouvoir central ou sa fragmentation annonciatrice d'anarchie. Aussi est-ce par raccroc que, en 1808, est entrevue l'existence d'une "Junte Centrale", mentionnée à seule fin de montrer qu'elle est indigne d'inspirer la confiance ; tout d'abord, elle a été dirigée par Floridablanca qui était " un véritable mannequin ", avant de tomber sous le contrôle de « Lorenzo Calvo, marchand épicier de Saragosse » et de "Tilly, autrefois condamné aux galères » (JP, 28 décembre 1808). En 1810, la «Junte de Séville » ou «Junte Suprême » 
est accusée de se livrer à un détestable despotisme qualifié, sans autre précision, de « révolutionnaire » (MU, 15 février 1810).

Une des façons de discréditer les nouvelles autorités résistantes consiste à prétendre que leur instauration illégitime ou désordonnée prépare une redoutable révolution de style français. C'est dans cette optique qu'est mentionnée la Régence en septembre 1808 :

«La Junte de Séville a pris le titre de Régence [...]. La prétendue Régence a publié des décrets qui paraissent copiés sur les arrêtés de salut public de Robespierre, et ont répandu la terreur dans toutes les provinces. De leur côté, les insurgés secondent de toute leur fureur la fureur de la Junte. » (JP, 29 septembre 1808)

Pour la raison probable que l'institution des Cortès donnerait à penser, fâcheusement, que la résistance populaire a fini par s'inscrire dans un cadre légal lui conférant une légitimité, il sera très peu question de la révolution libérale qui se développe à la Isla de León et à Cadix, sauf, pour les gazetiers, à reprendre la vieille antienne, à savoir que, là comme ailleurs dans le camp ennemi, les disputes ne cessent d'éclater (JE, 1er février 1812). En revanche, les députés, au lieu de montrer une activité débordante, paraissent voués à une « inaction léthargique » (JE, 26 mars 1812). Inutile donc d'attendre, même sous la forme de commentaires désobligeants, des allusions aux mesures adoptées par les députés gaditans dont on saura seulement, en vertu du leitmotiv anglophobe, qu'ils sont dirigés, sous cape, par le gouvernement allié dont «l'influence se fait sentir dans tous les actes des Juntes et des Cortès » (JE, 14 janvier 1813).

La première référence un peu substantielle à l'orientation politique des Cortès articule deux motifs de répulsion: leur assujettissement aux Anglais intrus et à un modèle révolutionnaire français éminemment redoutable:

«Wellesley, à force d'or et d'intrigue, a réuni à Cadix une soixantaine d'individus sans lieu, sans pain, sans mission, sous le titre pompeux de Cortès de l'Espagne ; les principes démagogiques et d'un véritable jacobinisme que ces forcenés ont professés depuis leur réunion, ont indigné les véritables Espagnols. » (MU, 20 mars 1811)

La régénération napoléonienne et le réformisme " joséphin »

50 La vaste entreprise de "désinformation " menée par une presse inféodée au pouvoir conduit les gazetiers à user sans parcimonie du grossier procédé de la dichotomie : le camp ennemi est celui de l'anarchie, de la révolution jacobine, de l'asservissement au gouvernement de Londres, de l'aveuglement, de la passion, du mensonge, de la déraison, de la régression, de l'immoralité..., tandis que le camp des Français et des « afrancesados » est celui de la vérité, de la raison et du salut.

51 Au long de la première moitié de 1808, les discours tenus par l'empereur, par Joseph et par les autorités espagnoles ralliées aux nouveaux détenteurs du pouvoir sont abondamment rapportés, puisqu'ils dessinent un avenir radieux pour l'Espagne. Dans cet ordre de choses, on trouve là la fameuse proclamation de Napoléon à Bayonne, le 25 mai, dans laquelle il se veut «le régénérateur de l'Espagne». On trouve là aussi le discours, tenu dans la même ville, par le «Président de la Junte " (JE, 15 juillet 1808). Moins connu est le texte, donné en espagnol et en français, de la proclamation de Joseph à Vitoria, le 12 juillet, dans laquelle le nouveau souverain reprend, quasiment à la lettre, le discours du «Président de la Junte » : parée de toutes les vertus, la nouvelle constitution assure au pays « la liberté civile et politique », « établit une représentation 
nationale » et «fait revivre nos anciens Cortès mieux organisés » (JE, 23 juillet 1808). Entre la mi-juin et la fin juillet, tous les discours, proclamations et adresses, tenus à Bayonne et dans les villes que traverse Joseph entre Bayonne et Madrid exposent l'importante mutation institutionnelle, de nature libérale (le terme « révolution » est banni) qui doit s'opérer avec l'aval d'une fraction considérable de la population (« hommes éclairés » et " propriétaires ») (MU, 5 septembre 1808).

Considérée ici en dehors des textes réglementaires adoptés à Bayonne, qui démentent éventuellement l'esprit ou réduisent la portée de cette révolution institutionnelle, celle-ci, telle qu'elle est présentée aux Français, se place sous la double égide des Lumières et du libéralisme, et se situe aux antipodes de la Révolution montagnarde ; elle a donc tout pour plaire à l'opinion publique française qui ne devrait avoir aucune raison de la détester ou de la craindre.

La presse impériale donne l'impression que le règne effectif de Joseph débute en juin 1808, coïncidant avec sa proclamation de Bayonne (MU, 28 juin 1808). Mais c'est seulement dans le $M U$ du 22 juillet qu'est livré le contenu de son programme réformateur, communiqué aux habitants de Vitoria, le 13 juillet. Le compte rendu détaillé de l'accueil chaleureux qui lui est réservé successivement à Miranda de Ebro et à Burgos ajoute l'idée que le nouveau souverain est admis par les autorités (l'archevêque, le chapitre, le "corregidor »), sinon par la population, dont on ne dit mot. De celle-ci il n'est question qu'à la mi-octobre : lors de son nouveau passage à Vitoria, le roi est entrevu à la promenade, au cours de laquelle il rencontre les habitants (MU, 8 octobre 1808). Les lecteurs français ignoreront jusqu'au bout que le nouveau roi est un roi à éclipses, tributaire des mouvements de progression ou de repli des troupes impériales. D'où l'impression curieuse que Joseph ne cesse de faire son entrée dans des villes où l'événement est marqué par des réceptions de délégations, des salves de canon, des sonneries de cloches, des illuminations de rues, des feux d'artifice, des messes et des Te Deum.

En 1809 et 1810, le roi donne l'impression, trompeuse ou forcée, qu'il règne véritablement ou, du moins, qu'il entend régner, ici jurant de « respecter l'unité de notre sainte religion et l'intégrité du territoire ", là visitant l'hôpital général de Madrid, à d'autres moments distribuant l' "Ordre royal et militaire d'Espagne", autorisant le retour dans leur couvent des religieux relevant des ordres supprimés, faisant ouvrir les portes du Jardin Botanique de Madrid «en présence d'une foule immense », se rendant au théâtre des Caños del Peral pour voir représenter une comédie de Calderón et « une sainete ». L'image que projette ainsi le roi Joseph est celle d'un monarque bien inspiré, modéré, indulgent, attentif au sort de ses sujets, aimant à se montrer, préférant le raisonnement à la force et, surtout, se campant en Espagnol, et non pas en frère de Napoléon.

Lors de la première réunion du Conseil d'État (cette appellation ne risque-t-elle pas de paraître excessivement française ?), il dévoile son rêve d'une Espagne forte et unie, pour ne pas dire unifiée et centralisée :

«L'Espagne sera invincible, indépendante, dès le jour même où le Castillan, l'Arragonais, le Basque, le Catalan, oubliant chacun ses anciennes divisions et les dénominations qui les ont perpétuées, confondra son nom avec celui d'Espagnol. » (MU, 19 mai 1809) 
56

A plusieurs reprises au cours de lannée 1809, le MU transcrit le contenu des décisions de nature variée qui, bien qu'appelées « décrets royaux », fortifient l'impression que, aux côtés du souverain, s'activent des ministres dévoués et compétents.

Auront ici valeur d'échantillons représentatifs d'une politique bien venue les décrets royaux suivants concernant l'ordre public, l'économie, le commerce, les finances, l'urbanisme, la justice et la vie culturelle: création d'une garde civique de 24 compagnies de 100 hommes chacune; "réformes salutaires» destinées au développement de l'agriculture le long des rives du Tage; suppression des bureaux de douane intérieure et transfert aux frontières; suppression de "la contribution dénommée Voto de San-Yago » (Santiago); à Madrid, ouverture d'avenues conduisant au palais royal, grâce à la démolition de couvents; abolition de la peine de potence et remplacement par la strangulation (sic); mise à l'écart de l'» Index établi par le cidevant tribunal de l'Inquisition» et censure seulement appliquée aux ouvrages offensants pour la religion d'état ou obscènes ou contenant des « maximes d'impiété ou de libertinage »; reprise des activités de la Société Madrilène des Amis du Pays, réactivation des spectacles théâtraux, célébration grandiose de la Fête du Pilar à Saragosse, mesures pour la conservation des monuments de Tolède...

Joseph, resté homme du XVIIIe siècle, se pose, bien qu'il n'en dise mot, en descendant de Charles III, et nullement en héritier de la Révolution française ou en disciple de son illustre frère. Alors qu'il est peut-être velléitaire ou subjugué par l'empereur, il est, aux yeux des lecteurs du $M U$, un monarque déterminé, éclairé, inventif, plein de bonnes intentions, désireux de gagner l'estime et l'amour de ses sujets et, surtout, impatient de voir la paix restaurée.

Les « afrancesados»

59 Avant que ne soit tiré de l'ombre le nom des proches collaborateurs de Joseph (ministres et conseillers), les lecteurs du $M U$ ont pu découvrir celui de plusieurs dignitaires qui, en 1808, ont donné l'impression, fondée ou non, de faire allégeance au nouveau souverain à Bayonne, puis le long de la route le conduisant vers sa nouvelle capitale. D'où l'apparition de noms nouveaux, souvent déformés, qui demeureront associés à l'» afrancesamiento »: Romero, Azanza, Pablo Arrivas (Arribas), Hermosillas (sic), Pereyra, Florente (Llorente), ducs de Frias, de Hijar, de Castelfranco, marquis d'Almenara, ou encore le nom, moins connu, de cet évêque de Zamora, Joaquin Mayoral, auteur d'une pastorale invitant les fidèles à la soumission :

«La guerre que la France nous a faite n'est point une guerre de religion; c'est un prétexte dont les Anglais se sont servis pour tromper et égarer le peuple.» (MU, 2 février 1809)

60 Sans doute parce que le nouveau souverain désire s'assurer l'appui du clergé séculier et opposer un démenti aux adversaires qui en font un ennemi de la religion et de l'Église, le $M U$ fait connaître plus volontiers les prises de position favorables des dignitaires ecclésiastiques que celles des Grands et des représentants de la société civile. D'où, par exemple, la transcription du discours prononcé par l'évêque auxiliaire de Saragosse, "S. Ander " (Miguel de Santander), "vieillard respectable par son âge et ses vertus ", qui ordonne aux prêtres de «ne pas se mêler des affaires d'État » et voue un culte au "Grand Napoléon », «l'homme qui semble être l'envoyé de Dieu sur la terre pour l'exécution des décrets de la Providence (MU, 14 mars 1809).

61 Malgré la répétition de ce genre de profession de foi, l'observateur d'aujourd'hui est encore loin de pouvoir reconstituer l'ensemble de l'argumentation échafaudée par les 
Espagnols - tous gens éclairés - ralliés aux nouvelles autorités ; à peine saura-t-on qu'y entrent, comme dans le discours cité plus haut, le providentialisme, l'admiration vouée à Napoléon, le désir d'une paix à obtenir au plus vite et l'espoir de réformes profondes mises en œuvre sans bouleversements sociaux.

Les deux Espagnes

Les appels des « afrancesados " engageant leurs compatriotes égarés à déposer les armes ou à ne pas les prendre en faveur des rebelles auraient suffi à suggérer que l'Espagne est scindée. D'un côté, vue de France, on a l'Espagne tragique, plongée dans la guerre, livrée aux insurgés et aux troupes anglaises qui terrorisent et maltraitent les habitants. De l'autre, on a l'Espagne dominée, certes sans ménagement, par les troupes impériales et placée, en principe, sous la bénéfique autorité du roi Joseph et de ses ministres. Entre 1809 et 1812, tentant de contrebalancer l'effet déplorable des innombrables récits de combats meurtriers, de scènes de pillage et de comportements indignes, les gazetiers ne lésinent pas sur les récits réconfortants de festivités organisées par les autorités «joséphines» et les militaires napoléoniens, et destinées à rendre les occupants tolérables, sinon aimables. On dirait ainsi que l'espoir renaît à tout moment, accompagnant le retour à une quasi-normalité du cours des vies. À Grenade, fin avril 1811, le général Sébastiani met la ville en fête avec feux d'artifice, courses de taureaux, soupers et bals au cours desquels «les dames, mises avec l'élégance et la recherche françaises, n'ont fait usage que d'étoffes françaises et espagnoles, puisqu'on n'a pas vu une seule parure provenant de l'Angleterre ou des colonies» (MU, 3 juin 1811). À Madrid, en 1812, « le Carnaval a été brillant » et « on a revu des mascarades disparues depuis 40 ans ». En juin de cette même année, à Gironne, "on a dansé le boléro, le fandango, et de jeunes personnes, aussi jolies qu'élégantes, ont fait admirer leurs grâces et leur souplesse dans l'exécution des danses nationales » (JE, 26 juin 1812). Mais impossible, malgré tout, d'oublier la guerre: quelques jours après cette information euphorisante, le $M U$ fait état d'une attaque conduite par les Français contre « le chef de bande Mina, fameux par ses rapines et ses cruautés ».

L' " heure de la vérité » sur la guerre d'Espagne a-t-elle sonné, aujourd'hui, pour les Français curieux de bien connaître ce long épisode? Pour les historiens, peut-être sonnera-t-elle lorsque les nombreux documents d'archives encore inexplorés à Paris auront été examinés dans leur totalité et lorsque ces informations seront venues compléter celles que les historiens espagnols, dans le cadre d'une histoire régionale et locale actuellement en plein renouveau, continuent à recueillir.

Dans cet « entre-deux chronologique » (1815 - début du XXIe siècle) vient se placer une quantité imposante de Mémoires autobiographiques émanant d'acteurs et de témoins, qui ont été publiés, les uns pendant la Restauration, les autres au cours de la seconde moitié du XIXe siècle, sans parler de ceux récemment exhumés ou réédités. D'intérêt et de portée inégaux, ces Mémoires livrent, au total, une vision originale et variée de la guerre d'Espagne, qu'il serait bon de confronter à celle offerte par la presse du Premier Empire, outrageusement tendancieuse et falsificatrice, comme on vient de le voir. Mais il faudrait se garder de penser que, d'un côté (les journaux) est le mensonge, et de l'autre (les Mémoires), la vérité dévoilée. En effet, cette littérature de témoignages est sujette à caution, dans la mesure où la plupart des auteurs, ou bien ont été de médiocres observateurs sur le terrain, ou bien s'emploient à se disculper après Waterloo, ou bien accablent, par représailles, les responsables de la sinistre « affaire espagnole » dans laquelle eux-mêmes ont été entraînés, ou bien se piquent de faire 
œuvre d'écrivains en cultivant, selon des proportions variables, l'horreur, le sensationnel, le pittoresque, les stéréotypes ou certaines composantes de la «Légende noire » de l'Espagne.

\section{NOTES}

1.Quand le sujet de cette étude a été envisagé, puis retenu, je n'avais pas encore pris connaissance de l'article consacré par Remedios Solano Rodríguez à « La Guerra de la Independencia española a través de Le Moniteur Universel (1808-1814) », article publié dans les Mélanges de la Casa de Velázquez, Madrid, t.XXXI (3), 1995, pp.55-75. J’y renvoie les lecteurs. Dans la mesure du possible, j'ai fait en sorte que les deux articles se complètent ; et, du reste, on observera que l'historienne espagnole s'est bornée au seul Moniteur Universel, tandis que j'ai travaillé sur un corpus plus vaste. Il n'y a pas, en fin de compte, de contradictions entre les deux approches. Mon présent travail prolonge celui qui a donné lieu à une communication sous le titre de : « La propaganda francesa sobre la intervención en España en 1808 », Séminaire « Los franceses en Madrid, 1808 : Información, Propaganda y Comportamiento popular ", à l'occasion du colloque organisé par la Asociación para el Estudio de la Guerra de la Independencia, Madrid, 26, 27 et 28 mai 2003.

2.Cf. le répertoire établi par Jean Tulard, Nouvelle bibliographie critique des mémoires sur l'époque napoléonienne (...), Genève, Librairie Droz, 1991.

3.On songe par exemple à l' Histoire de la Guerre d'Espagne et de Portugal de 1807 à 1814, par M.Sarrazin, maréchal de camp, Paris, J.G.Dentu, Imprimeur-Libraire, 1814.

4.Cabanis (André), La presse sous le Consulat et l'Empire (1799-1814), Paris, Société des études robespierristes, 1975.

5.On utilisera par la suite les sigles suivants :-MU pour Le Moniteur Universel-JE pour le Journal de l'Empire-JP pour le Journal de Paris-PU pour Le Publiciste.

6.Dans Lettres inédites de Napoléon 1er (1800-1815), éditées par Léon Lecestre, Paris, 1897, t.I, p.194.

7.On ne sait trop quel terme utiliser : « gazetier » évoque trop l'Ancien Régime, et « rédacteur » et « journaliste » semblent bien modernes...

8.Signe de l'intérêt généralisé que suscite la laine espagnole est la publication, annoncée dans le MU du 20 octobre 1811, de l'Essai sur les mérinos de M. Giron de Buzaraingues.

9.Cf. mon article « La batalla de Somosierra. La inmediata versión oficial », dans Francisco Javier Pastor Muñoz - María Jesús, El campo de batalla de Somosierra (30XI-1808), Consejería de las Artes de la Comunidad de Madrid, nº10, Madrid, 2001, pp. $121-125$ 


\section{RÉSUMÉS}

Compte tenu des impératifs rigoureux de la propagande officielle, la tournure de plus en plus inquiétante prise par les événements dans la Péninsule entre 1808 et 1814 interdit de faire tourner à la gloire des militaires napoléoniens l'intervention au sud des Pyrénées. Celle-ci a tout d'abord été justifiée publiquement par la décision de l'empereur d'œuvrer à la "régénération » du pays. Plus tard, la guerre est présentée comme menée contre les Anglais et contre des rebelles indigènes rabaissés à la catégorie de bandits méprisables. La presse impériale, étroitement contrôlée, recourt à de grossiers procédés d'occultation ou de déformation des faits de guerre, ajoutée à l'ignorance de la révolution politique entreprise par les patriotes libéraux à Cadix. Malgré les défaites de Baylen, des Arapiles et de Vitoria, les lecteurs sont invités à penser que les soldats napoléoniens sont les victimes des éprouvantes conditions météorologiques, de la pénurie en hommes et en matériel, et enfin de la conduite, par les insurgés, d'une guerre irrégulière (la guérilla) que les Français ne parviennent pas à maîtriser.

\section{The Peninsular War in the Imperial Press (1808-1814)}

Given the tough constraints of official propaganda, the dismal turn of events in the Peninsula between 1808 and 1814 was hardly a commendation for the Napoleonic military in their intervention south of the Pyrenees. The initial public justification was the Emperor's decision to work toward the "regeneration" of the country. Later, the war was said to be waged against the English and against native rebels disparaged as despicable bandits. The imperial press, under tight control, resorted to brazen falsifications or distorsions of the facts of war, added to ignorance of the political revolution undertaken by the liberal patriots in Cadiz. Despite the defeats of Baylen, the Arapiles and Vitoria, readers were asked to swallow the line that Napoleon's soldiers were the victims of the inclement weather, shortage in manpower and equipment, and finally the conduct by insurgents of an irregular (guerilla) war which the French were unable to control.

\section{INDEX}

Mots-clés : Espagne, guérilla, afrancesados, désinformation, insurgés, légende noire, occupation

\section{AUTEUR}

JEAN-RENÉ AYMES

Université Paris III - Sorbonne Nouvelle 